\title{
La comédie en France dans les années 1630: des auteurs en quête de légitimité
}

\section{Sandrine Berregard}

\section{(2) OpenEdition}

1 Journals

\section{Édition électronique}

URL : https://journals.openedition.org/studifrancesi/25891

DOI : 10.4000/studifrancesi.25891

ISSN : 2421-5856

Éditeur

Rosenberg \& Sellier

\section{Édition imprimée}

Date de publication : 1 avril 2007

Pagination : 107-116

ISSN : 0039-2944

\section{Référence électronique}

Sandrine Berregard, "La comédie en France dans les années 1630: des auteurs en quête de

légitimité », Studi Francesi [En ligne], 151 (LI | I) | 2007, mis en ligne le 30 novembre 2015, consulté le 22 novembre 2021. URL : http://journals.openedition.org/studifrancesi/25891 ; DOI : https://doi.org/ 10.4000/studifrancesi.25891

\section{(c) (i) (9)}

Studi Francesi è distribuita con Licenza Creative Commons Attribuzione - Non commerciale - Non opere derivate 4.0 Internazionale. 


\section{La comédie en France dans les années 1630: des auteurs en quête de légitimité}

Le renouveau des principaux genres dramatiques, qui marque la période des années 1630 en France, s'accompagne d'une série de déclarations qui témoignent de la volonté des auteurs de mettre à distance les modèles hérités du passé. «La nouveauté de ce genre de Comédie, dont il n'y a point d'exemple en aucune Langue, et le style naif, qui faisait une peinture de la conversation des honnêtes gens, furent sans doute cause de ce bonheur surprenant, qui fit alors tant de bruit» ${ }^{1}$ : comme le rappellera l'examen de Mélite, la comédie se transforme profondément, notamment sous l'influence de Corneille, qui exprime son intention de renoncer aux personnages-types de la comédie à l'italienne en vue de fonder un nouveau type de comédie, qui soit la représentation la plus réaliste possible de la société contemporaine ${ }^{2}$. De nouveaux modèles voient le jour, qui illustrent également les relations étroites qu'entretiennent alors entre eux les différents genres (comédie pastorale, comédie romanesque, etc.): la comédie se décline sous diverses formes, et les expressions ne manquent pas pour témoigner de l'extraordinaire richesse du genre à un moment décisif de son évolution.

Cependant, les théoriciens du théâtre, soucieux de conserver à la tragédie une supériorité que la hiérarchie traditionnelle des genres lui reconnaît depuis toujours, peinent à donner de la comédie une définition susceptible de servir de cadre théorique aux futurs dramaturges. Ce sont donc les auteurs de comédies eux-mêmes (Corneille, mais aussi Rotrou, Mairet) qui dans leurs épîtres dédicatoires, préfaces, avertissements au lecteur, etc. élaborent au fil des pages une sorte de théorie du genre dont leurs pièces sont censées être une illustration. C'est précisément à cet ensemble de péritextes ${ }^{3}$ que nous voudrions porter notre attention, non seulement pour voir quelle théorie du genre se constitue et sur quels fondements elle repose, mais aussi et surtout pour examiner les éventuelles tensions entre théorie et pratique. La voie paraît en effet étroite entre le désir de faire reconnaître la comédie comme un genre respectable et la nécessité de ne pas lui attribuer exactement les mêmes mérites qu'à la tragédie, genre que les intéressés eux-mêmes pratiquent par ailleurs ${ }^{4}$. Le débat qui s'engage autour de la question du respect des règles dès la fin des années 1620 explique aussi que la production de ces textes, situés à la lisière entre théorie et pratique, soit si abondante en cette période-là. Leur hétérogénéité même montre enfin qu'il est

(1) Mélite, examen dans Euvres complètes t. 1, éd. critique de G. Couton, Paris, Gallimard (Pléiade), 1980, pp. 5-6.

(2) «On n'avait jamais vu jusques-là que la Comédie fît rire sans Personnages ridicules, tels que les Valets bouffons, les Parasites, les Capitans, les Docteurs, etc.» (ibid., examen, p. 6). Du moins les premières comédies de Corneille offrent-elles de la jeune bourgeoisie parisienne une peinture conforme à la réalité du temps.
(3) Au sujet du péritexte en général, voir en particulier P. Lane, La Périphérie du texte, Paris, Nathan, (Linguistique), 1992 et G. GenETtE, Seuils, Paris, 1987.

(4) Ainsi, comme il le rappelle dans l'épître dédicatoire des Galanteries du duc d'Ossonne, Mairet fut d'abord un auteur de tragédies (Théatre du XVII siècle t. 1, éd. critique de J. Scherer, Paris, Gallimard, Pléiade, 1975, p. 596). 
diverses manières de prendre position sur des sujets aussi déterminants que celui-ci: qu'un Rotrou par exemple choisisse de dédier sa Bague de l'oubli au roi n'est pas seulement à interpréter comme un acte de déférence à l'égard du plus puissant, mais doit aussi être entendu comme une tentative de réhabiliter un genre dévalorisé. De telles initiatives se révèlent d'autant plus déterminantes que, suivant une tradition héritée de la Poétique d'Aristote à laquelle manquent les chapitres consacrés à la comédie, les théoriciens ne lui accordent en général qu'une faible valeur, voyant au contraire dans la tragédie un genre codifié et par là même respectable 6 . Malgré leur désir de voir la comédie se conformer aux mêmes règles que la tragédie, les auteurs s'efforcent de montrer sa capacité à exister indépendamment des autres genres dramatiques. Sans nier la spécificité de la comédie, qui reste (et doit rester) un genre essentiellement divertissant, ils tentent donc de lui donner une légitimité à travers une série de déclarations destinées à la faire admettre comme un genre sérieux et respectable, notamment auprès des doctes.

Le corpus sur lequel portera l'analyse comprend, outre les premières comédies de Corneille ${ }^{7}$, Les Visionnaires de Desmarets de Saint-Sorlin, Les Galanteries du duc d'Ossonne de Mairet et, enfin, La Bague de l'oubli et La Belle Alphrède de Rotrou', dont les dates de publication s'échelonnent de 1633 à 1639. Malgré son caractère nécessairement un peu arbitraire, ce choix porte sur un ensemble de pièces qui reflètent les principaux aspects de la comédie durant cette période. L'hétérogénéité même du corpus témoigne de la diversité extrême du genre, dont les particularités sont d'autant plus difficiles à saisir qu'il s'apparente parfois à d'autres genres (tragicomédie et pastorale principalement) ${ }^{9}$. Les textes qui accompagnent les pièces de Corneille présentent en outre une caractéristique majeure, qu'il n'est sans doute pas inutile de rappeler, à savoir que les péritextes d'origine (préfaces, avertissements au lecteur, épîtres dédicatoires) sont complétés par des examens composés une trentaine d'années plus tard. Cet écart chronologique permet de suivre le parcours d'un auteur qui, après s'être presque entièrement voué à la comédie, se tourne vers d'autres genres dramatiques et qui, à l'occasion de la réédition de ses Euvres complètes en 1660, corrige ses premières pièces pour les adapter aux nouvelles attentes du public. Rotrou lui-même, à l'époque où il crée ses premières comédies, pratique aussi tragédie et tragi-comédie ${ }^{10}$; et la relative indifférenciation des genres dramatiques telle qu'elle s'illustre dans son théâtre ${ }^{11}$ montre à quel point la comédie est devenue un genre aux contours incertains.

(5) Rotrou, La Bague de l'oubli, «Au roi», dans ibid., p. 731.

(6) Il est évident que la noblesse des personnages, alliée à la noblesse de la langue, a permis à la tragédie d'être considérée comme le genre dramatique le plus prestigieux.

(7) Mélite (1633), La Veuve (1634) La Place royale (1637), La Galerie du Palais (1637), La Suivante (1637) et L'Illusion comique (1639). Les dates indiquées entre parenthèses correspondent aux premières publications.

(8) Mélite, p. 1-89, La Veuve ou le traître trabi, p. 199-298, La Galerie du Palais ou l'amie rivale, p. 299-381, La Suivante, p. 383-459, La Place royale ou l'amoureux extravagant, p. 467-531 et L'Illusion comique, p. 611-688 dans Euvres complètes t. 1, éd. cit.; Desmarets de Saint-Sorlin, Les Visionnaires, p. 405-492 dans Théâtre du XVII siècle t. 2, éd. cit., 1986; MaIRET, Les Galanteries du duc d'Ossonne, p. 595-668, Rotrou, La Bague de l'oubli, p. 731-791 et La Belle Alphrède, p. 793-864 dans ibid. t. 1.

(9) Ainsi, les premières comédies de Corneille empruntent à la pastorale son schéma dramatique (la chaîne des amours non-partagées).

(10) Du moins si on se réfère aux dates de publication, puisque dans le cas de Rotrou il est très difficile de préciser les dates de création. Voir à ce sujet P. Gethner, «La chronologie du théâtre de Rotrou. Mise au point», p. 242-257 dans Revue d'bistoire du théâtre III, 1991, n 171.

(11) Voir à ce sujet J. Morel, Jean Rotrou dramaturge de l'ambiguité, Paris, Colin, 1968, pp. 136-137. 


\section{La mise en ceuvre d'une véritable stratégie}

Si on se reporte aux textes théoriques publiés entre la fin du seizième et le début du siècle suivant, on constate que la comédie est rarement définie pour elle-même: opposée à la tragédie ou comparée à la farce, elle peine à faire reconnaître sa spécificité $^{12}$. Aussi, pour dire ce qu'est la comédie, Laudun d'Aigaliers par exemple, dans son Art poétique français paru en 1597, juge nécessaire de prendre la tragédie pour modèle de référence:

Le commencement de la Tragédie est joyeux et la fin est triste; le commencement de la comédie est triste, et la fin est joyeuse: Les paroles et carmes de la Tragédie sont graves, et ceux de la comédie sont légers, et ne traitent que de risée: Les personnages de la tragédie sont habillés somptueusement et ceux de la comédie sont habillés communément. Anciennement les personnages de la Tragédie avaient les cothurnes, c'est-à-dire des brodequins ou souliers qui venaient jusques à mi-jambes, qui étaient fort somptueux, et ceux de la comédie avaient un soc, qui est une sorte de souliers, qui n'est pas si somptueuse ${ }^{13}$.

S'inspirant des rares modèles théoriques issus de l'Antiquité ${ }^{14}$, minimales et simplificatrices, les définitions qui en sont généralement données montrent qu'en cette toute fin du seizième siècle la comédie est loin d'avoir acquis ses lettres de noblesse. Quant aux théoriciens des années 1630-1640, ils s'intéressent principalement à la tragédie, qu'ils jugent en général supérieure à la comédie. C’est ainsi que dans les premières pages de sa Poétique (1640) La Mesnardière, après avoir traité successivement des genres sérieux que sont la tragédie et la tragi-comédie, exprime ouvertement le mépris que lui inspire la comédie: «La deuxième espèce de notre Poème Dramatique est la simple Comédie, fort recherchée autrefois par la populace de Grèce, et par celle d'Italie, et même encore approuvée par des nations fort sérieuses, bien qu'elle n'ait rien de grave, de noble, ni de magnifique» ${ }^{15}$. Reste donc aux auteurs eux-mêmes à faire valoir les mérites d'un genre qu'à l'occasion ils n'hésitent pas à rapprocher de la tragédie, tel Corneille qui explique le choix du titre donné à La Galerie du Palais en invoquant les exemples de Sophocle et d'Euripide ${ }^{16}$, ou Mairet qui pour justifier sa pratique des deux genres emprunte à Pline le Jeune la métaphore des deux maisons ${ }^{17}$. Même s'ils affirment donc ne pas se prendre trop au sérieux, les auteurs de comédies entendent bien que leurs pièces ne soient pas considérées avec mépris.

Face à une telle situation, la première attitude possible pour ceux qui choisissent de s'essayer au genre est celle qui consiste à afficher une (apparente) désinvolture. Les auteurs s'excusent en effet volontiers de la (prétendue) médiocrité de leurs pièces, comme le montre par exemple l'épître «comique et familière» que Mairet adresse à

(12) C'est ainsi que, dans son dictionnaire publié en 1573, Nicot distingue les deux grands types de comédie qui existent dans le théâtre latin: «Comédie et farce, Comoedia, Fabula. Comédies esquelles sont introduits personnages de bas état et du commun, Tabernariae comoediae. Comédies esquelles était traité des affaires des anciens rois et magistrats, qui usaient d'une manière de robe qu'on appelait praetexta, Praetextae comediae» (Nicot, Trésor de la langue française tant ancienne que moderne, 1573).

(13) LaUdun D'Aigaliers, Art poétique français divisé en cinq livres, 1re éd. 1597 , livre $V$ chap. 5 «De la différence de la tragédie et comédie», p. 206-207 dans éd. réalisée sous la dir. de J.-C.

\section{Monferran, Paris, S.T.F.M, 2000.}

(14) Selon la définition d'Aristote, la comédie est «une imitation d'hommes sans grande vertu» (Poétique V, trad. de M. Magnien, Paris, Le Livre de Poche, 1990, p. 91).

(15) La Poétique, Paris, Sommaville, 1640, Slatkine reprints 1972 , chap. 2 «Division du poème dramatique en ses espèces», p. 7. D'Aubignac dans sa Pratique du théatre (1657) affiche la même condescendance (voir passim).

(16) La Galerie du Palais, «À Madame de Liancourt», p. 301.

(17) Les Galanteries du duc d'Ossonne, « À très docte et très ingénieux Antoine Brun», p. 598. 
Antoine Brun, «procureur général au parlement de Dole» ${ }^{18}$, ou encore la lettre que Corneille écrit à Mme de Liancourt pour lui offrir sa Galerie du Palais et dans laquelle il exprime ses craintes à l'idée que la pièce ne soit pas digne de celle à qui elle est destinée $^{19}$. Aussi la comédie est-elle le plus souvent définie comme un simple «divertissement $\gg^{20}$ : tel est le mot qu'emploient, par exemple, Rotrou dans l'épître dédicatoire de sa Belle Alphrède et Corneille dans la lettre qu'il adresse à Mme de Liancourt à propos de La Galerie du Palais ${ }^{21}$. Le mot apparaît encore une fois sous sa plume dans l'avertissement au lecteur de La Veuve et donne lieu à une définition de la comédie qui témoigne de la transformation du genre en ce début des années 1630:

Son ornement n'est pas dans l'éclat des vers. C'est une belle chose que de les faire puissants et majestueux, cette pompe ravit d'ordinaire les esprits, et pour le moins les éblouit, mais il faut que les sujets en fassent naitre les occasions, autrement c'est en faire Parade mal à propos, et pour gagner le nom de Poète perdre celui de judicieux. La Comédie n'est qu'un portrait de nos actions, et de nos discours, et la perfection des portraits consiste en la ressemblance. Sur cette maxime je tâche de ne mettre en la bouche de mes acteurs, que ce que diraient vraisemblablement en leur place ceux qu'ils représentent, et de les faire discourir en honnêtes gens, et non pas en Auteurs ${ }^{22}$.

En même temps qu'il reconnaît la modestie de son projet («La Comédie n'est $q u^{\prime} u n$ portrait ...» $)^{23}$, l'auteur formule à propos de la comédie une exigence de réalisme et de vraisemblance, qui en l'éloignant des stéréotypes de la farce ou de la commedia dell'arte lui confère une réelle dignité24. Il explique ainsi qu'il a emprunté à la «vieille Comédie» le «Personnage de la Nourrice», mais qu'il l'a métamorphosé en «celui de la Suivante» ${ }^{25}$, qui elle-même deviendra le personnage principal d'une autre de ses pièces. Néanmoins, la simplicité du style qui caractérise la comédie («... les faire discourir en honnêtes gens»), et que la nature même des sujets réclame ${ }^{26}$, l'empêche de rivaliser avec la tragédie et l'idéal de perfection qu'elle incarne.

Comme le suggèrent les exemples qui viennent d'être cités, le choix du dédicataire (lorsqu'il existe) participe lui-même de la stratégie mise en œuvre par les auteurs pour tenter de faire reconnaître à la comédie un sérieux dont elle peut désormais se prévaloir. Offrir sa pièce à un haut personnage, c'est en effet non seulement le moyen d'obtenir une aide souvent indispensable, mais c'est aussi et surtout une manière de conférer au genre une certaine noblesse. Aussi les pièces sont-elles presque toutes dédiées à de grands seigneurs: Mélite et La Galerie du Palais le sont respectivement

(18) Ibid., p. 595. Si Mairet conçoit cette épître à l'image de la pièce qu'elle est chargée d'introduire, Corneille remarque que la préface de La Veuve est «déjà trop longue pour une comédie» (p. 203).

(19) La Galerie du Palais, «À Madame de Liancourt», p. 301.

(20) Comme le rappelle Mairet, le théâtre est devenu «le divertissement du prince et de son principal ministre» (Les Galanteries du duc d'Ossonne, «A très docte et très ingénieux Antoine Brun», p. 596). Rotrou ne dit pas autre chose lorsque, dans l'épître dédicatoire de La Bague de l'oubli, il affirme que la comédie (au sens de pièce de théâtre) «se peut vanter d'être [...] le divertissement même de [Sa] Majesté» (p. 731).

(21) La Galerie du Palais, «À Madame de Liancourt», p. 301 .

(22) Ibid., «Au lecteur», p. 202.
(23) Nous soulignons.

(24) Josse Bade considérait déjà que, quoique fictif, le sujet de la comédie devait être vraisemblable (cité dans l'éd. critique de l'Art poétique français de Laudun d'Aigaliers, p. 192 n. 20). Dans l'examen de La Veuve, l'exigence de vraisemblance est une nouvelle fois formulée: «Il ne pouvait être qu'environ Midi quand il [Alcidon] en a formé le dessein, puisque Célidan venait de ramener Clarice (ce que vraisemblablement il a fait le plus tôt qu'il a pu, ayant un intérêt d'amour qui le presse de lui rendre ce service en faveur de son Amant)» (p. 217).

(25) Ibid., examen, p. 304.

(26) C'est ainsi que, dans l'examen de La Veuve, Corneille reconnaît que «le style n'est pas plus élevé ici que dans Mélite» (p. 218), pièce à propos de laquelle il affirmait déjà que «[sa] façon d'écrire [était] simple et familière» (Mélite, «Au lecteur», p. 4). 
à M. et Mme de Liancourt, La Veuve à Mme de la Maisonfort ${ }^{27}$ et Les Galanteries du duc d'Ossonne à «Antoine Brun, procureur général au parlement de Dole» ${ }^{28}$. Quant à La Bague de l'oubli, elle est destinée au personnage le plus puissant du royaume - un choix qui s'explique également par la présence dans la pièce du personnage d'Alfonse, roi de Sicile, encore que celui-ci se voie privé de son autorité. Dans tous les cas, le dédicataire apparaît comme une figure idéale, à laquelle l'ensemble du lectorat est susceptible de s'identifier ${ }^{29}$, signe que la comédie est devenue un genre sérieux, capable de toucher les plus raffinés d'entre les lecteurs.

Il est un autre moyen par lequel les auteurs s'efforcent de donner au genre un sérieux qui jusqu'à présent lui a presque toujours été refusé: la nécessité de se conformer à la règle des trois unités. Malgré sa réticence à les appliquer dans toute leur rigueur, Corneille juge chacune de ses pièces à la lumière de ce qui est pour les théoriciens du classicisme un des principes fondateurs du genre tragique. Encore, selon son propre aveu, cette exigence n'est-elle à l'époque de Mélite, et alors que les revendications des Réguliers commencent tout juste à se faire entendre, que le fruit de son intuition ${ }^{30}$. Si cette préoccupation se manifeste surtout dans les examens de 1660, on la voit cependant déjà s'exprimer dans l'avis au lecteur de La Veuve, pièce pour laquelle l'auteur explique qu'il lui a fallu trouver une sorte de compromis entre l'extrême rigueur et la trop grande liberté $e^{31}$. Les premières comédies de Corneille et les commentaires qui les accompagnent témoignent ainsi de la souplesse avec laquelle l'auteur s'est efforcé d'appliquer la règle des trois unités: il explique, par exemple dans l'examen de Mélite, qu'il est parvenu à élaborer un système astucieux, qui rapproche la durée de l'action de celle de la représentation ${ }^{32}$ tout en utilisant les espaces libres laissés par les entractes ${ }^{33}$; et quand bien même la règle des vingt-quatre heures ne serait pas respectée, il importe que les actes soient proportionnés les uns aux autres, comme l'illustrent $L a$ Veuve et La Galerie du Palais, dont les cinq actes correspondent à cinq jours consécutifs ${ }^{34}$. De même, soucieux de respecter strictement la règle de l'unité de lieu, l'auteur a dû user de certains procédés qui parfois l'ont conduit à sacrifier le principe de vraisemblance. Ainsi, dans La Galerie du Palais, Célidée et Hippolyte, dont les maisons se font face, se confient leurs secrets sur le pas de leur porte, alors qu'il eût été plus vraisemblable de les voir se rencontrer dans une chambre ou un salon ${ }^{35}$. L'auteur rappelle qu'il s'agit là d'une convention, que les Anciens connaissaient déjà et que les spectateurs modernes, ceux de 1660 plus encore que ceux de 1630, acceptent sans peine ${ }^{36}$. Or, telle est à peu de choses près la remarque que fera Corneille à propos

(27) À propos de ces personnages, voir les notes de G. Couton, p. 1157-1158, 1253-1254 et 1289.

(28) Les Galanteries du duc d'Ossonne, «À très docte et très ingénieux Antoine Brun», p. 595. L'auteur conçoit cette dédicace comme un «acte de gratitude et de reconnaissance» envers un homme qui, dit-il, a été à l'origine de sa carrière d'écrivain (ibid.). Comme le rappelle Mairet, la pièce devait être dédiée au duc de Montmorency (p. 596), mais la mort de ce dernier obligea le poète à se choisir un autre dédicataire.

(29) La Galerie du Palais, «Â Madame de Liancourt», p. 301. Ainsi, l'absence de Mme de Liancourt aux représentations de La Galerie du Palais empêcha l'auteur de profiter pleinement des «acclamations publiques» que lui valut la pièce (ibid.).

(30) Mélite, examen, p. 5.

(31) La Veuve, «Au lecteur», p. 203.

(32) Voir à ce sujet Discours des trois unités, d'action, de jour et de lieu: «La représentation dure deux heures, et ressemblerait parfaitement, si l'action qu'elle représente n'en demandait pas davantage pour sa réalité. Ainsi ne nous arrêtons point ni aux douze ni aux vingt-quatre heures, mais resserrons l'action du poème dans la moindre durée qu'il nous sera possible, afin que sa représentation ressemble mieux, et soit plus parfaite» (Euvres complètes t. 3, éd. cit., 1987, p. 184).

(33) Mélite, examen, p. 8. C'est également ce que dit Corneille à propos de La Suivante: «Le temps n'en est pas plus long que celui de la représentation, si vous en exceptez l'heure du dîner qui se passe entre le premier et le second Acte» (épître dédicatoire, p. 387).

(34) La Veuve, examen, p. 216 et La Galerie du Palais, examen, p. 304.

(35) Ibid., p. 302-303.

(36) Ibid., p. 303. C'est précisément au nom du principe de vraisemblance qu'il place une partie de l'acte III de La Place royale dans le cabinet d'Angélique (examen, p. 472). 
d'Horace dans son Discours de la tragédie $e^{37}$ - preuve que du point de vue de la dispositio les deux genres ne diffèrent désormais guère l'un de l'autre, du moins dans le respect des règles propres à la dramaturgie classique. Le titre que l'auteur choisit de donner à La Place royale ou à $\mathrm{La}$ Galerie du Palais témoigne encore de son souci de faire coïncider (autant que possible) unité de lieu et lieu unique, même si par la suite les scènes ne se déroulent pas toutes au même endroit ${ }^{38}$. De la même manière, Corneille s'efforce de respecter la règle de l'unité d'action: comme il l'explique, il mêle dans la plupart de ses comédies au moins deux intrigues, qui néanmoins ne sauraient se développer indépendamment l'une de l'autre ${ }^{39}$. Enfin, il manifeste la volonté de respecter le principe des bienséances, citant pour exemple la tragédie, dont la vocation première est d'édifier le spectateur: c'est ainsi qu'à propos de La Place royale il dénonce, tout autant que l'«inégalité de mœurs» de son héros, l'amour excessif d'Angélique qui ne craint pas d'être enlevée par son amant malgré le comportement étrange de ce dernier ${ }^{40}$. Desmarets manifeste la même préoccupation lorsque, dans son argument (qui n'est autre en réalité qu'un avertissement au lecteur) ${ }^{41}$, il démontre la cohérence de l'intrigue en dépit du nombre important des personnages qui s'y rencontrent ${ }^{42}$. En s'efforçant ainsi d'observer la règle des trois unités, les auteurs expriment leur fidélité à une tradition héritée de l'Antiquité et, par ce moyen, confèrent à la comédie une dignité presque égale à celle qui est habituellement reconnue à la seule tragédie. Toutefois, ils refusent d'appliquer aveuglément les préceptes édictés par les Anciens. Dans l'épître dédicatoire de La Suivante, Corneille estime ainsi qu'il serait inopportun de reprocher aux auteurs modernes de ne pas suivre à la lettre des règles que leurs aînés ne respectaient pas toujours ${ }^{43}$. Sachant, ajoute-t-il, qu'une stricte observation des règles édictées par Aristote et Horace ne suffit nullement à garantir la qualité et le succès d'une pièce, il affirme la nécessité de les adapter à la nature du sujet ${ }^{44}$. Dans d'autres cas en revanche, il se montre extrêmement pointilleux, à telle enseigne qu'il signale des régularités ou des irrégularités qui en 1630 pouvaient passer inaperçues. Ainsi, dans son examen de La Galerie du Palais, tous les détails de la pièce sont passés au crible et toutes les entorses aux règles de la dramaturgie classique soigneusement notées. Sans doute Corneille tient-il à faire la preuve de sa bonne volonté à l'égard des doctes, dont le jugement est de nature à déterminer le succès ou l'échec d'une pièce.

\section{Des auteurs face à leurs propres contradictions}

Dans un tel contexte, il est difficile de définir la comédie sans la comparer à la tragédie. Sur ce point, les auteurs sont cependant contraints d'adopter une attitude nuancée: d'un côté en effet, il ne s'agit pas pour eux de conférer à la comédie le même degré de dignité que celui qui est habituellement reconnu à la seule tragé-

(37) «L'unité de lieu y est assez exacte, tout se passe dans une salle. Mais si on en faisait un roman avec les mêmes particularités de scène en scène, que j'y ai employées, ferait-on tout passer dans cette salle?...» (Discours de la tragédie, dans Euvres complètes, t. 3, p. 164).

(38) La Place royale, examen, p. 471-472; La Galerie du Palais, examen, p. 302-303.

(39) Toutefois, à propos de La Place royale, il remarque une «duplicité d'action» (examen, p. 471). (40) Ibid.

(41) L'argument au sens strict consiste à faire un résumé de l'intrigue.

(42) Les Visionnaires, argument, p. 406: «Toutes ces folies, bien que différentes, ne font ensemble qu'un sujet».

(43) Ibid.: «Je vous laisse donc à penser si notre présomption ne serait pas ridicule, de prétendre qu'une exacte censure ne pût mordre sur nos ouvrages, puisque ceux de ces grands génies de l'Antiquité ne se peuvent pas soutenir contre un rigoureux examen».

(44) Ibid., p. 387: «Savoir les règles, et entendre le secret de les apprivoiser adroitement avec notre Théâtre, ce sont deux sciences bien différentes, et peut-être que pour faire maintenant réussir une pièce, ce n'est pas assez d'avoir étudié dans les livres d'Aristote et d'Horace». 
die; mais d'un autre côté, il convient d'assurer au genre une certaine respectabilité. Cette double exigence se manifeste particulièrement dans l'épître dédicatoire des Galanteries du duc d'Ossonne, puisque Mairet rapproche sa première comédie de ses précédentes pièces, La Silvanire, La Sophonisbe et la Virginie, respectivement pastorale, tragédie et tragi-comédie, tout en rappelant que les attentes du lecteur ne sont pas de même nature dans une comédie et dans une pièce sérieuse ${ }^{45}$. Il manifeste une certaine désinvolture en se moquant des honneurs habituellement rendus aux auteurs dramatiques $^{46}$, mais affiche également de hautes ambitions et prétend faire partie de ceux qui ont modernisé le théâtre ${ }^{47}$. Cette double attitude est celle de la plupart des auteurs de comédies en ce début des années 1630: sans se prendre trop au sérieux, comme l'exige la nature même des sujets, ils souhaitent faire de la comédie un genre éminemment respectable. Toutefois, ce n'est pas la position qu'ils adoptent tous: ainsi Rotrou, qui emprunte bon nombre de ses sujets à la comedia espagnole ${ }^{48}$ et dont le genre de prédilection est la tragi-comédie $e^{49}$, ne se préoccupe guère de la conformité de ses pièces à la règle des trois unités. Il est vrai que le caractère polymorphe du genre offre aux auteurs une liberté à laquelle la tragédie par exemple ne permet guère d'accéder, comme l'illustre encore L'Illusion comique de Corneille, qui selon le point de vue adopté sera considérée comme une pièce parfaitement régulière ou parfaitement irrégulière. Face à de tels écarts, il semble difficile de construire à propos de la comédie une théorie du genre qui soit réellement homogène à l'exemple de celle qui a pu être élaborée à propos de la tragédie.

Quelle que soit leur position à l'égard des règles, les auteurs de comédies se montrent tous soucieux de plaire au public. Néanmoins, un partage assez net se fait entre ceux qui ont à cœur de satisfaire toutes les catégories de spectateurs et ceux qui à l'inverse visent un public restreint. Corneille entend ainsi s'adresser à la Cour et au peuple tout autant qu'aux doctes, mais s'empresse d'ajouter qu'il faut avant tout «[gagner] la voix publique» et que, si une pièce régulière ne reçoit pas l'approbation de l'ensemble des spectateurs, les savants eux-mêmes attribueront cet échec à une mauvaise compréhension des règles ${ }^{50}$. C'est dire le peu de crédit que l'auteur accorde aux doctes et aux principes qu'ils édictent. Ainsi sans doute s'explique l'originalité d'une pièce comme Les Galanteries du duc d'Ossonne: il s'agit en effet d'une comédie d'intrigue qui enfreint le principe des bienséances ${ }^{51}$ et reste totalement étrangère aux modèles hérités de l'Antiquité. À la différence de ses contemporains, l'auteur des $V i$ sionnaires indique précisément la nature du public auquel il entend destiner sa pièce: le «divertissement honnête» qu'il propose s'adresse à d' «honnêtes gens» ${ }^{52}$, qui seuls selon lui sont capables d'apprécier ses qualités ${ }^{53}$.

(45) Les Galanteries du duc d'Ossonne, «À très docte et très ingénieux Antoine Brun», p. 597-598.

(46) «ll est vrai qu'on nous fait au Louvre des sacrifices de louanges et de fumées, comme si nous étions les Dieux de l'Antiquité les plus délicats, où nous aurions besoin qu'on nous traitât plus grossièrement et qu'on nous offrît plutôt de bonnes hécatombes de Poissy, avec une large effusion de vin d'Arbois, de Beaune et de Condrieu» (ibid., p. 596).

(47) Ibid.

(48) Il rappelle ainsi que le sujet de La Bague de l'oubli est emprunté à Lope de Vega.

(49) Sur un total de trente-cinq pièces on compte dix-sept tragi-comédies.

(50) La Suivante, épître dédicatoire, p. 387-388: «Il faut, s'il se peut, y ajouter les règles, afin de ne déplaire pas aux Savants, et recevoir un applau- dissement universel, mais surtout gagnons la voie publique: autrement, notre pièce aura beau être régulière, si elle est sifflée au Théâtre, les Savants n'oseront se déclarer en notre faveur, et aimeront mieux dire que nous aurons mal entendu les règles, que de nous donner des louanges quand nous serons décriés, par le consentement général de ceux qui ne voient la Comédie que pour se divertir».

(51) Voir à ce sujet la notice de J. Scherer, éd. cit., p. 1272-1273.

(52) Voir la définition du mot Honnête dans le Dictionnaire universel de Furetière (1690): «Ce qui mérite de l'estime, de la louange, à cause qu'il est raisonnable, selon les bonnes mœurs» (t. II, réimpr. Paris, Le Robert, 1978).

(53) Ce propos est en substance celui de Rotrou dans l'épître dédicatoire de La Bague de l'oubli 
Les péritextes définissent donc une sorte de modèle théorique, qui cependant n'est pas toujours en accord avec la réalité des textes. Ainsi, bien que dans l'examen de Mélite Corneille prétende avoir renoncé aux personnages de la commedia dell'arte, le genre tel qu'il renaît en cette fin des années 1620 n'est pas étranger à cette tradition, comme le montrent la figure de Matamore dans L'Illusion comique ou encore les personnages de Desmarets, dont la plupart sont d'une manière ou d'une autre hérités de la comédie à l'italienne $e^{54}$. Les auteurs justifient les raisons de leur choix en expliquant qu'ils ont aussi cherché à s'éloigner de la tradition: selon l'expression de Corneille, le capitan, «inventé exprès pour faire rire» ${ }^{55}$, ne joue dans l'intrigue qu'un rôle secondaire; et lorsque dans son argument il dresse le portrait de chacun de ses personnages, Desmarets manifeste la volonté de les adapter à la réalité contemporaine ${ }^{56}$.

Praticiens avant d'être théoriciens, les auteurs théorisent a posteriori, et pourtant leurs réalisations pratiques ne concordent pas toujours avec la manière dont ils conçoivent le genre. Corneille rêve ainsi d'une comédie qui soit parfaitement régulière, sans pour autant atteindre toujours à cet idéal. Il semble donc que les péritextes aient notamment pour fonction d'orienter la lecture des pièces dans le sens voulu par l'auteur: ainsi, malgré la complexité du langage dans certaines de ses scènes ${ }^{57}$, il n'est pas sûr que le lecteur des Visionnaires par exemple y voie une pièce destinée à un public choisi. De la même manière, la présence du soldat fanfaron dans L'Illusion comique semble faire de la comédie de Corneille une pièce capable de toucher un large public, alors même que son auteur s'attache à mettre en avant la complexité de son architecture ${ }^{58}$.

Il est un autre élément qui révèle l'ambiguïté de la position adoptée par les auteurs: certains de leurs dédicataires sont à peu près impossibles à identifier, soit parce que leurs noms sont dissimulés, soit tout simplement parce qu'ils n'existent pas - preuve que la comédie n'est pas encore prête à renoncer au caractère un peu fantaisiste qui la distingue des autres genres, en particulier de la tragédie. Ainsi en est-il de la mystérieuse Sylvie à qui Rotrou dédie sa Belle Alphrède, de Monsieur**** à qui s'adresse Corneille au seuil de La Place royale et, enfin, de Mlle M.F.D.R. qui se voit offrir L'Illusion comique. Qu'il s'agisse de dédicataires réels ou fictifs, chacun d'eux illustre d'une certaine manière un des principaux aspects de la pièce qu'il se charge ainsi d'introduire: le choix du prénom de Sylvie est probablement dû à la coloration pastorale de l'intrigue, tandis que l'usage de l'astéronyme vise sans doute à éviter tout risque de confusion avec un héros dont le comportement à l'égard de la gent féminine est peu recommandable ${ }^{59}$.

À travers ces différentes formes de péritexte, il semble donc bien que les auteurs cherchent à s'immiscer dans le processus de lecture. Ainsi, lorsque Corneille met en avant l'originalité de L'Illusion comique, il tend à faire oublier la part des conventions qui pourtant occupent dans la pièce une place importante. En 1660, au moment où il

à propos du théâtre en général: «Puisque enfin la comédie est en un point où les plus honnêtes récréations ne lui peuvent plus causer d'envie ...» («Au roi», p. 731).

(54) Le modèle italien continuera cependant d'inspirer les auteurs de comédies, comme le montre par exemple Le Parasite de Tristan L'Hermite (1653) - preuve de leur difficulté à se départir d'une certaine tradition.

(55) L'Illusion comique, examen, p. 614.

(56) Les Visionnaires, argument, p. 405-406. Il en est ainsi notamment de l'«amoureuse d'Alexandre le Grand», dont l'imagination se nourrit des ro- mans héroïques, nombreux dans la première moitié du XVII ${ }^{e}$ siècle, et de l'«amoureuse de la Comédie», qui fréquente assidûment le théâtre.

(57) Voir par exemple le monologue d'Amidor $(\mathrm{I}, 3)$, qui regorge de références mythologiques.

(58) «Voici un étrange monstre que je vous dédie. Le premier Acte n'est qu'un Prologue, les trois suivants sont une Comédie imparfaite, le dernier est une Tragédie, et tout cela cousu ensemble fait une Comédie» (L'Illusion comique, «À Mademoiselle M.F.D.R.», p. 614).

(59) La Place royale, « À Monsieur***», p. 470. 
donne de son Théâtre une nouvelle édition assortie de ses trois Discours sur le poème dramatique, l'auteur apparaît désireux de montrer la cohérence de son œuvre. Rallié au parti des Réguliers comme la plupart de ses contemporains et jouissant désormais d'une renommée flatteuse, il s'érige en censeur et les principes qu'il édicte, ses pièces (y compris ses premières comédies) sont supposées s'y conformer. Ainsi rassemblés, les péritextes déterminent une stratégie complexe, par laquelle les auteurs espèrent pouvoir à distance maîtriser leurs lecteurs. Toutefois, ces interventions se révèlent plus ou moins contraignantes pour le lecteur: ainsi Corneille, qui agit successivement sur le lectorat de 1630 et sur celui de 1660, élabore pour chacune de ses pièces un discours qui s'adapte aux attentes du moment (si sa position à l'égard des règles se veut d'abord assez souple, elle se radicalise ensuite sous l'influence des théoriciens). En revanche, Mairet et plus encore Rotrou laissent à leurs lecteurs une grande liberté d'interprétation: tandis que le premier traite la comédie avec la légèreté qui lui convient, le second se contente de présenter brièvement chacune de ses pièces. Par leur diversité même, ces attitudes témoignent de la disparité du genre, qui décidément risque de rendre difficile l'élaboration d'une théorie capable de faire reconnaître la comédie comme un genre réellement constitué. Qu'y a-t-il en effet de fondamentalement commun entre les pièces de Rotrou, qui aussi bien du point de l'inventio que du point de la vue de la dispositio ressemblent à des tragi-comédies autant qu'à des comédies, et celles de Corneille, par lesquelles l'auteur s'efforce d'adapter le modèle pastoral aux exigences d'un public urbain? Du reste, Rotrou lui-même s'est conformé, dans certaines de ses autres comédies, au «modèle cornélien ${ }^{60}$, sans pour autant toujours respecter la règle de l'unité de lieu dont son contemporain ne cesse pourtant de rappeler la nécessité.

«Comédie sérieuse»: une contradiction dans les termes? Ce sont les modèles latin et italien auxquels les auteurs se disent prêts à renoncer. Les successeurs de Hardy inventent un nouveau concept, celui de «divertissement honnête», suggérant ainsi que la comédie est capable de plaire à un public raffiné, sans pour autant lui faire perdre sa vocation première, qui est de divertir plus que d'édifier. Aussi cet ensemble de péritextes montre combien est incertain le statut générique de la comédie telle qu'elle se développe dans les années 1630. En cette période de bouleversements, les auteurs s'emploient à créer de nouvelles formes, mais il leur est assurément difficile de faire table rase du passé - d'où la nécessité de diriger le parcours de lecture, d'orienter le regard du lecteur vers les aspects qui leur semblent les plus novateurs et surtout les plus à même de donner au genre une respectabilité jusqu'alors inaccessible. Ainsi sans doute s'explique l'écart qui parfois sépare les pièces elles-mêmes des textes qui les accompagnent, les déclarations d'intention de la réalité du genre. Avertissements au lecteur, préfaces, épîtres dédicatoires ne sont d'ailleurs pas les seules formes de péritexte à témoigner des difficultés auxquelles sont confrontés les auteurs dans leur quête de légitimité: titres, sous-titres, arguments révèlent également la nature de leurs choix en même temps que les limites de leur entreprise ${ }^{61}$. Finalement, c'est sans doute

(60) Tel est le cas notamment des comédies pastorales que sont La Clorinde, Le Filandre, La Céliane et La Florimonde.

(61) Ainsi, par leur complexité même, les argu- ments qui accompagnent respectivement Mélite et La Veuve montrent que ces pièces relèvent de la comédie d'intrigue. 
en définissant la comédie comme un genre moyen que les auteurs parviendront à résoudre leurs propres contradictions, avant que la «grande comédie» de caractère ne s'impose avec Molière quelques décennies plus tard. Néanmoins, le dictionnaire de Furetière, publié dans les dernières années du siècle, témoigne encore de la diversité des sens attachés au mot puisque, après avoir défini la comédie comme une «pièce de théâtre composée avec art, en prose, ou en vers», il en indique l'acception la plus usuelle («Se prend plus particulièrement pour les pièces qui représentent des choses agréables et non sanglantes, et des personnes de médiocre condition»), pour rappeler in fine le lien qui unit la comédie à la tradition farcesque («Se dit encore en un sens plus étroit, pour une farce, une facétie, où on n'introduit guère que des valets et des bouffons, pour dire des choses plaisantes, et faire rire» $)^{62}$. 